\title{
Block diagonalization precoding and power allocation for clustering small-cell networks
}

\author{
Toha Ardi Nugraha ${ }^{1}$, Indar Surahmat ${ }^{2}$, Firdaus ${ }^{3}$ \\ ${ }^{1,2}$ Department of Electrical Engineering, Universitas Muhammadiyah Yogyakarta, Indonesia \\ ${ }^{3}$ Department of Electrical Engineering, Universitas Islam Indonesia, Indonesia
}

\begin{tabular}{l} 
Article Info \\
\hline Article history: \\
Received Dec 21, 2019 \\
Revised Mar 15, 2020 \\
Accepted Apr 9, 2020 \\
\hline Keywords: \\
Block diagonalization \\
Cellular network \\
Clustering network \\
Power allocation \\
Precoding \\
Small-cells
\end{tabular}

\section{Article history:}

Received Dec 21, 2019

Revised Mar 15, 2020

Accepted Apr 9, 2020

\section{Keywords:}

Cellular network

Clustering network

Precoding

\begin{abstract}
The clustering network is a solution to improve data-rate transmission in small-cells. In this case, clustering small-cells (CSCs) adopt a multiple antennas concept. The multiple antennas are used to maximize the downlink data-rate transmission at the users, but it requires precoding techniques to minimize interference among CSC users. This paper proposes a block diagonalization (BD) as a precoding technique for minimizing interference among CSC users. The performance of the BD precoding implemented on the clustering network under various numbers of small-cells. The CSC also implements a water-filling power allocation (PA-CoopWF) to distribute the available transmission power along with the CSCs antennas. To show the performance, our paper simulates two types of precoding techniques; those are the proposed BD and minimum mean square error (MMSE) in CSCs. Based on the receiver user parts under the overlapping coordination of CSCs, our method based on the BD precoding achieves considerably higher data-rate transmission compared to the MMSE precoding, especially on larger clusters. The simulation also shows that by implementing CSC with the $\mathrm{BD}$ in short-range distances and higher numbers of antennas, it promotes better data-rate performances compared to the MMSE precoding by 2.75 times at distance $100 \mathrm{~m}$ and $67 \%$ at 50 antennas.
\end{abstract}

This is an open access article under the CC BY-SA license.

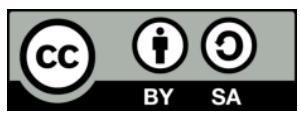

\section{Corresponding Author:}

Toha Ardi Nugraha,

Department of Electrical Engineering,

Universitas Muhammadiyah Yogyakarta,

Jl. Brawijaya, Tamantirto, Kasihan, Bantul, Yogyakarta 55183, Indonesia.

Email: toha@ft.umy.ac.id

\section{INTRODUCTION}

The implementation of small-cells is one of the effective solutions to improve data-rate in cellular networks [1]. Small-cells are also a solution to extend cellular coverage. Small-cells are commonly installed in urban or indoor building areas, so small-cells access-point is connected via cable backhaul to the gateway. In [2], the authors propose an architecture of small-cells based resource allocation for wireless backhaul in two-tier heterogeneous networks. The authors only focus on the backhaul transmission on the clustered small-cells. Small-cells are also allowed to exchange their channel state information (CSI) over the gateway [3,4]. Therefore, the architecture of small-cells enables easy implementation of a clustering network. There are some researchs in clustering focused on the small-cells network [5,6]. The main concept of clustering networks is that some cells in a network coordinates each other [7]. This method manages coordination information for improving the performance of communication [8]. Clustered small-cells is one of the solutions in order to improve the data-rate, particularly for the user in the cell edge, which has interference effect from the neighbor small-cells $[9,10]$. The coordination method is one of the key 
technologies to improve the data-rate of cell-edge users [11]. The interference problem is exploited or mitigated by the coordination method between small-cells [12]. The coordination method improves the efficiency of radio resources as well as satisfying the requirements of high data-rate, improve spectral and energy efficiency [13-15].

One of the solutions to improve data-rate in a network is by employing multiple-input and output (MIMO) concepts. MIMO concepts manage the frequency-time resources from transmitters to receivers [16]. On the other hand, interference problems appear in this multi-user MIMO concept. Therefore, a precoding technique is a scheme to guarantee orthogonality across parallel channels and suppress interference before the transmission signal. There are some techniques for employing precoding techniques, namely linear and non-linear. A linear precoding is described by standalone matrix, for example, zero-forcing $(\mathrm{ZF})[17,18]$ and minimum mean square error (MMSE) $[19,20]$. The benefit of the linear precoding is the low complexity in computations. In [21], the authors proposed a multi-user MIMO precoding technique in order to reduce the negative impact of co-tier interference in the heterogeneous network. In [22], the authors investigated a network of small-cells where the BSs were ready to form multiple clusters and coordinate to maximize the overall sum-rate. The authors used a multi-user multiple antennas precoding based zero-forcing beamforming (ZFBF) schemes. However, ZF and MMSE precodings are restricted only with one of the receiver antennas. The antenna receiver cannot manage interference itself and only receive one spatial channel. In this case, the transmitter must perform precodings in order to suppress the inter-user interference. In [23], the authors proposed a regularized channel inversion (CI) precoding technique to enhance the sum-rate assuming only single-antenna users are available in the system. In [24], the authors proposed a novel of cooperative block diagonalization (BD) precoding to eliminate inter-user interference users under the error model on the CSI. Therefore, the BD precoding is a great solution to be implemented in the clustering and multi-user systems.

This paper proposes clustering small-cells (CSCs) using the BD precoding in order to manage interference between the users in order to improve the data transmission rate of the networks. This paper also investigates the impact of the additional number of antennas and the increment of distance between the CSCs and the users on the performance. Moreover, the effect of changing the number of antennas is also analyzed. This research assumes that CSCs use different channels with BS; therefore, there is no interference between small-cells and BS users. In order to show the performance, our proposed algorithm is compared with other linear precoding, MMSE techniques [25]. The rest of the paper is organized as follows: In section 2, we describe the system model. Section 3 investigates our proposed method that is the interference mitigation and the power allocation method. Section 4 explains the result and discussion of the simulations. Finally, section 5 explains the conclusion of this paper.

\section{SYSTEM MODEL}

In this paper, we consider a various number of CSCs in a network as an example shown in Figure 1. This paper investigates massive small-cells using transmit antennas $N_{t}$ and $u_{j}$ user equipments (UEs) with the number of users $j=1 \ldots N$, and receiver antennas having $N_{r}$. In this case, each transmitter $N_{t}$ is equipped with two antennas and each receiver $N_{r}$ is also equipped with two antennas, and also the number of CSCs is with three small-cells. The users are randomly located at the cell edge zone and within the overlapping of the neighbor cells. This system assumes that each small-cells has the same frequency. The rest of the main simulation parameters are shown in Table 1

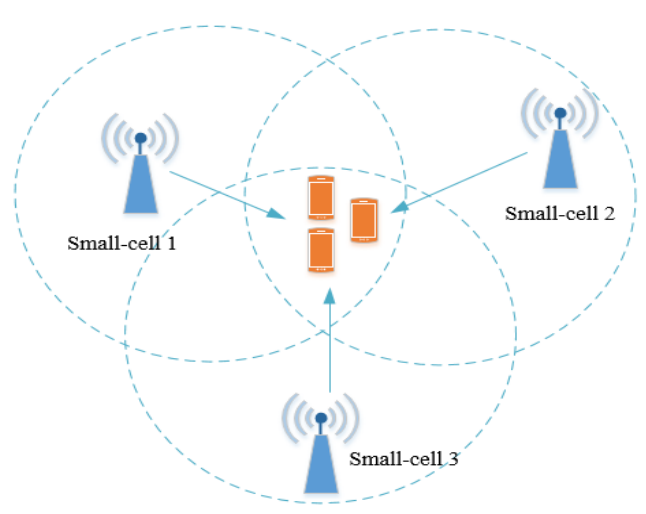

Table 1. Simulation parameters

\begin{tabular}{lc}
\hline \multicolumn{1}{c}{ Parameter } & Value \\
\hline Carrier frequency & $2 \mathrm{GHz}$ \\
System bandwidth & $5 \mathrm{MHz}$ \\
Transmission Power & $23 \mathrm{dBm}$ \\
Noise Spectral density & $-174 \mathrm{dBm} / \mathrm{Hz}$ \\
No. of antenna each SC & 2 antennas \\
No. of antenna each user & 2 antennas \\
Pathloss model & ITU indoor \\
Antenna pattern & Omni directional \\
\hline
\end{tabular}

Figure 1. Clustering small-cells with coordination method 


\section{PROPOSED METHOD}

This paper proposes CSC in order to improve the data-rate of the user in the cell edge. This scheme mitigates the inter small-cells interference because the coordination in CSC facilitates a sharing of channel feedback between the small-cells over a gateway. As illustrated in Figure 2, the proposed algorithm is performed in three stages, each associated with exchanging the coordination request, coordination response, the last stage is a sending the channel feedback and transmission of the data.

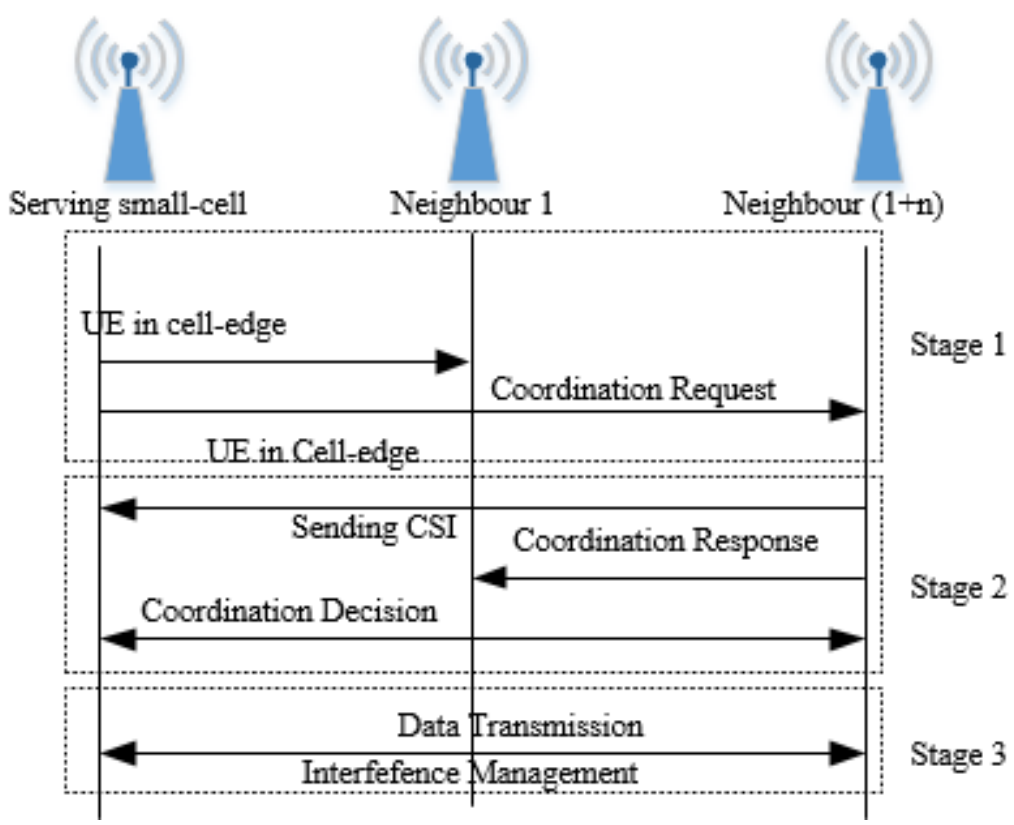

Figure 2. Stage of coordination methods of clustering small-cells, contain serving and neighbor small-cells

- Stage 1, this stage is a coordination REQUEST that user $u_{j}$ is monitored at the cell edge of serving smallcells. The user $u_{j}$ uses the channel gain $H$ without considering the quality of the channels; therefore, there is inter-cell interference between small-cells and neighboring small-cells. If the serving small-cells increase the transmission power, the SNR of the cell edge user $u_{j}$ might be lost.

- Stage 2, in this stage, the method of CSCs coordinates their neighbors for making CSCs. First, we calculate the number of CSCs antennas. The equation is formulated by $N_{t}=\sum_{i=1}^{C} n_{t, i}$ where the number of CSCs containing serving and neighbor small-cells is $C$. Second, the total number of receiver antennas $u_{j}$ is calculated by $N_{r}=\sum_{i=1}^{C} n_{r, u_{j}}$. Therefore, the channel matrix to receiver user $u_{j}$ is given by $H_{u_{j}}=\left[\begin{array}{lll}H_{u_{j}}^{1} \ldots & H_{u_{j}}^{C}\end{array}\right]$. Third, the channel matrix of the multiple antennas for user $u_{j}$ given by $H_{u_{j}}=$ $\left[\begin{array}{lll}H_{u_{1}}^{H} \ldots & H_{N}^{H}\end{array}\right]^{H}$ where $H$ superscript with $i=1 \cdots N$ is the conjugate transpose of a channel matrix. And last, the channel matrix at the user $u_{j}$ is calculated by

$$
y_{u_{j}}=\sum_{i=1}^{C} H_{u_{j}}^{i} w_{u_{j}}^{i} u_{u_{j}}^{i}+\sum_{i=1}^{C} H_{u_{j}}^{i} \sum_{u_{n \neq u_{j}}} w_{u_{n}}^{i} u_{u_{n}}^{i}+n_{u_{j}}
$$

$H_{u_{j}}^{i}$ is the channel matrix from small-cells $i$ to user $u_{j}, \sum_{u_{n \neq u_{j}}} w_{u_{n}}^{i} u_{u_{n}}^{i}$ represents the inter small-cells interference experienced by user $u_{j}, w_{u_{n}}^{i}$ is explained in the next section, and $n_{u_{j}}$ is complex gaussian noise entries with zero-mean and variances $\sigma_{n}^{2}$. The interference between small-cells may lose its diversity gain and the precoding technique manages inter small-cells interference nulling for CSCs. Therefore, the objective of this clustering RESPONSE step is to ensure that the interference CSCs are optimally managed. This step is used to find $S i \ldots N$ from the neighboring serving small-cells. $S$ is the serving small-cell and $N$ is the number of neighbor small-cells. Neigbour small-cells are used for 
clustering if all off them can reach to the cell edge user. Then, the CSC is generated with $C=S i$...N. To manage this step, CSI is informed to the gateway of small-cell networks. Therefore, all access-points small-cells exchange information to each other and make DECISION.

- Stage 3, this stage is used to manage the data transmission rate of cell-edge users. The data transmission rate of the user in the cell-edge is managed without reducing the quality of the CSCs. Inter user interference management is performed by employing a BD precoding technique and it is described in the next section.

\subsection{Interference management}

In this section, the proposed scheme implements the BD precoding in CSCs. Strengthening the earlier statement, the BD precoding is used for mitigating inter-user interference in CSCs. The SNR for the user $u_{j}$ with the BD precoding under perfect CSI is as follow:

$$
y_{u_{j}}=\sum_{i=1}^{C} H_{u_{j}}^{i} w_{u_{j}}^{i} u_{u_{j}}^{i}+n_{u_{j}} \quad \text { for all } j \neq n
$$

In order to obtain $w_{u_{j}}^{i}$, at the first, we must define $H_{u_{j}}^{i}$ from

$$
H_{u_{j}}^{*}=\left[\left(H_{1}\right)^{H} \ldots\left(H_{u_{j}-1}\right)^{H}\left(H_{u_{j}+1}\right)^{H} \ldots\left(H_{N}\right)^{H}\right]^{H}
$$

and singular value decomposition (SVD) to decompose the channel matrix into parallel non interference spatial layers. The channel matrix is calculated by

$$
S V D\left[H_{u_{j}}^{*}\right]=U_{u_{j}}^{*}\left[\begin{array}{ll}
V_{m, u_{j}}^{*} & 0
\end{array}\right]\left[\begin{array}{ll}
S_{u_{j}}^{*(1)} & S_{u_{j}}^{*(0)}
\end{array}\right]^{H}
$$

$V_{m, u_{j}}^{*}$ is a diagonal matrix with non-zero elements devote sub-channel's gain. $S_{u_{j}}^{*(1)}$ and $S_{u_{j}}^{*(0)}$ are composed of vectors that are corresponding to zero singular and non-zero singular values. Thus signals from other users are not received.

\subsection{Power allocation}

In this paper, we use a power allocation (PA) using coordination water-filling (CoopWF) [26]. PA-CoopWF is a technique used to distribute the total available power along with the various antennas based on the SNR distributions. The PA-CoopWA gains a better performance for the coordination channel compared with the equal power for small-cells. The PA-CoopWF is calculated as follows:

$$
P_{u_{j}}=\mu-\frac{\sigma^{2}}{L u_{j}}=\frac{1}{N}\left\{P_{c}+\sum_{u_{j}=1}^{N} \frac{\sigma^{2}}{L u_{j}}\right\}-\frac{\sigma^{2}}{L u_{j}}
$$

$P_{c}$ is coordination power CSCs and $L u_{j}$ is path-loss propagation for user $u_{j}$. Finally, the formula to get the data-rate $R_{u_{j}}$ for the user $u_{j}$ using the PA-CoopWF is as follows:

$$
R_{u_{j}}=\sum_{u_{j}=1}^{N} B \log _{2}\left[1+\frac{P_{u_{j}} H_{u_{j}}^{*}}{\sigma^{2}}\right]
$$

\section{RESULTS AND DISCUSSION}

This section describes the simulation results of the proposed methods and compared it to the previous works. Figure 3 shows that the performance of proposed methods is slightly better almost $10 \mathrm{Mbps}$ compared to MMSE investigated in single small-cells of each SNR target. The simulation also investigated a number of CSCs in each SNR target. As an example, the CSCs with $N_{t}=4,6$, and 8 antennas, 
our proposed scheme improves sum-rate about 10\%, 21\%, and 40\% compared to MMSE with the same number of antennas, respectively. Since the MMSE precoding technique works without removing the noise of each channel, the channel interference cannot be mitigated perfectly at the receiver parts.

Figure 4 shows a comparison of the proposed method and MMSE in the same massive-antennas deployment on CSCs scenarios. Based on the simulations, the graph informs that the average data-rate improves gradually with the increase of the number of antennas and the performance gap of the proposed precoding technique becomes larger in comparison to the MMSE methods. As an example, the simulations investigate with 50 and 100 antennas; the proposed method improves the average-rate about $67 \%$ and $90 \%$ compared to the MMSE precoding technique, respectively.

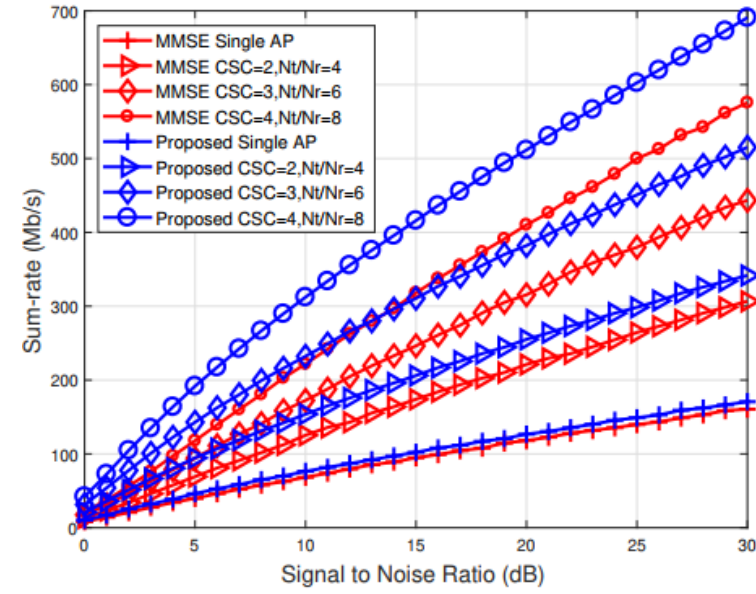

Figure 3. The impact of CSCs with difference level of SNR receivers

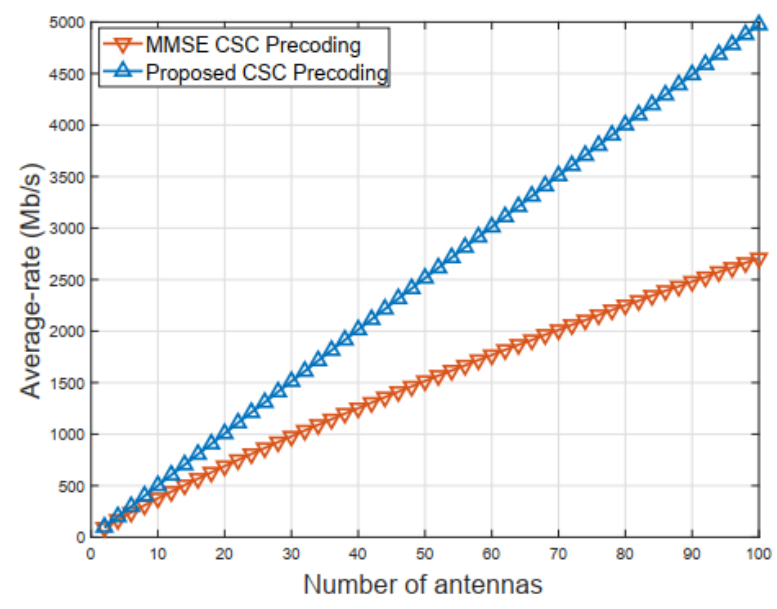

Figure 4. BD and MMSE precoding technique with various numbers of antennas

Finally, Figure 5 shows the average-rate when the CSCs and users are evaluated by the distances. The proposed methods archive an average data-rate gain about 2.75 times compared to the MMSE precoding at $100 \mathrm{~m}$. The graph shows that even though the average data-rate drops proportionally with the increment of the distance, the performance gap of the proposed precoding technique in comparison to the MMSE methods becomes larger. For the case that the distance of the receiver user fixed at $500 \mathrm{~m}$ from the transmitter of CSC, the proposed method provides a better transmission rate about 5 times compared to the MMSE precoding technique. Therefore, our proposed precoding scheme is more efficient.

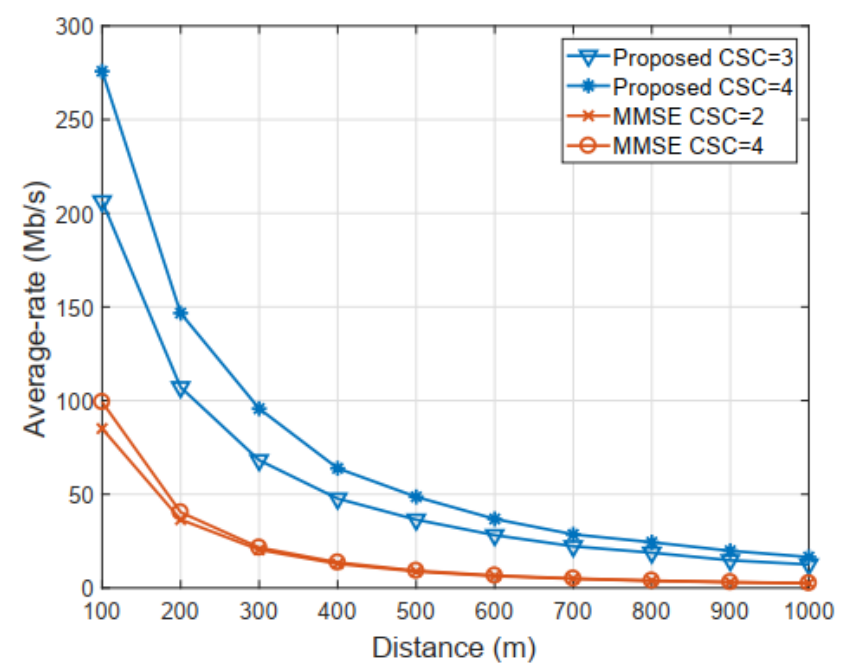

Figure 5. Downlink data-rate with difference distance from CSCs transmitter 


\section{CONCLUSION}

This paper investigates the clustering small-cells and precoding technique for improving data-rate in the networks. The clustering small-cells methods employ the MIMO concept and implement a precoding technique to minimize interference. This paper formulates clustering small-cells contains multiple antennas for maximizing the downlink data-rate for the users. The BD precoding is proposed in clustering small-cells to mitigate the interference channel between the users. This research also implements a water-filling power allocation to improve the gain of each channel. Simulation results show that our proposed algorithms obtain better performance of data rate compared to the MMSE precoding technique by $67 \%$ at 50 antennas and 2.75 times at distance $100 \mathrm{~m}$. For future research, our research will investigate the implementation of small-cells in an indoor environment with channel error problems. Our future algorithm will also improve the precoding technique to optimize the impact of imperfect channel state information.

\section{ACKNOWLEDGEMENTS}

This research is supported by the Indonesia Endowment Fund for Education (LPDP), Ministry of Research, Technology, and Higher Education of Republic Indonesia, and Universitas Muhammadiyah Yogyakarta.

\section{REFERENCES}

[1] C. Wang et al., "Cellular architecture and key technologies for 5G wireless communication networks," in IEEE Communications Magazine, vol. 52, no. 2, pp. 122-130, Feb 2014.

[2] W. Hao and S. Yang, "Small Cell Cluster-Based Resource Allocation for Wireless Backhaul in Two-Tier Heterogeneous Networks With Massive MIMO," in IEEE Transactions on Vehicular Technology, vol. 67, no. 1, pp. 509-523, Jan. 2018.

[3] D. Lopez-Perez, I. Guvenc, G. de la Roche, M. Kountouris, T. Q. S. Quek and J. Zhang, "Enhanced intercell interference coordination challenges in heterogeneous networks," in IEEE Wireless Communications, vol. 18, no. 3, pp. 22-30, June 2011.

[4] R. Yao, Y. Liu, L. Lu, G. Y. Li and A. Maaref, "Cooperative Precoding for Cognitive Transmission in Two-Tier Networks," in IEEE Transactions on Communications, vol. 64, no. 4, pp. 1423-1436, April 2016.

[5] C. T. K. Ng and H. Huang, "Linear Precoding in Cooperative MIMO Cellular Networks with Limited Coordination Clusters," in IEEE Journal on Selected Areas in Communications, vol. 28, no. 9, pp. 1446-1454, December 2010.

[6] S. Bassoy, H. Farooq, M. A. Imran and A. Imran, "Coordinated Multi-Point Clustering Schemes: A Survey," in IEEE Communications Surveys \& Tutorials, vol. 19, no. 2, pp. 743-764, Secondquarter 2017.

[7] R. Seno, T. Ohtsuki, W. Jiang, and Y. Takatori, "A low-complexity cell clustering algorithm in dense small cell networks," EURASIP Journal on Wireless Communications and Networking, vol. 2016, no. 1, pp. 1-11, 2016.

[8] E. Pateromichelakis, M. Shariat, A. Quddus, M. Dianati and R. Tafazolli, "Dynamic Clustering Framework for Multi-Cell Scheduling in Dense Small Cell Networks," in IEEE Communications Letters, vol. 17, no. 9, pp. 1802-1805, Sep 2013.

[9] Z. Chen, J. Lee, T. Q. S. Quek and M. Kountouris, "Cooperative Caching and Transmission Design in ClusterCentric Small Cell Networks," in IEEE Transactions on Wireless Communications, vol. 16, no. 5, pp. 3401-3415, May 2017.

[10] V. Jungnickel et al., "The role of small cells, coordinated multipoint, and massive MIMO in 5G," in IEEE Communications Magazine, vol. 52, no. 5, pp. 44-51, May 2014.

[11] S. Ni, J. Zhao, H. H. Yang, T. Q. S. Quek and Y. Gong, "Small Cell Range Expansion with Interference Mitigation for Downlink Massive MIMO HetNets," 2018 IEEE Global Communications Conference GLOBECOM, Abu Dhabi, United Arab Emirates, pp. 1-7, 2018.

[12] R. Irmer et al., "Coordinated multipoint: Concepts, performance, and field trial results," in IEEE Communications Magazine, vol. 49, no. 2, pp. 102-111, Feb 2011.

[13] M. O. Al-Kadri, A. Aijaz and A. Nallanathan, "Ergodic Capacity of Interference Coordinated HetNet with FullDuplex Small Cells," Proceedings of European Wireless 2015; 21th European Wireless Conference, Budapest, Hungary, pp. 1-6, 2015.

[14] E. Björnson, L. Sanguinetti and M. Kountouris, "Deploying Dense Networks for Maximal Energy Efficiency: Small Cells Meet Massive MIMO," in IEEE Journal on Selected Areas in Communications, vol. 34, no. 4, pp. 832-847, April 2016.

[15] E. Björnson, M. Kountouris and M. Debbah, "Massive MIMO and small cells: Improving energy efficiency by optimal soft-cell coordination," ICT 2013, Casablanca, pp. 1-5, 2013.

[16] E. Castañeda, A. Silva, A. Gameiro and M. Kountouris, "An Overview on Resource Allocation Techniques for Multi-User MIMO Systems," in IEEE Communications Surveys \& Tutorials, vol. 19, no. 1, pp. 239-284, Firstquarter 2017.

[17] L. D. Nguyen, T. Q. Duong, H. Q. Ngo and K. Tourki, "Energy Efficiency in Cell-Free Massive MIMO with Zero-Forcing Precoding Design," in IEEE Communications Letters, vol. 21, no. 8, pp. 1871-1874, Aug. 2017.

[18] Q. Vu, L. Tran, R. Farrell and E. Hong, "Energy-Efficient Zero-Forcing Precoding Design for Small-Cell Networks," in IEEE Transactions on Communications, vol. 64, no. 2, pp. 790-804, Feb. 2016. 
[19] L. D. Nguyen, H. D. Tuan, T. Q. Duong, O. A. Dobre and H. V. Poor, "Downlink Beamforming for EnergyEfficient Heterogeneous Networks With Massive MIMO and Small Cells," in IEEE Transactions on Wireless Communications, vol. 17, no. 5, pp. 3386-3400, May 2018.

[20] D. Ying, H. Yang, T. L. Marzetta and D. J. Love, "Heterogeneous Massive MIMO with Small Cells," 2016 IEEE 83rd Vehicular Technology Conference VTC Spring, Nanjing, pp. 1-5, 2016.

[21] E. Driouch, W. Ajib, and C. Assi, "Power control and clustering in heterogeneous cellular networks," Wireless Networks, vol. 23, no. 8, pp. 2509-2520, 2017.

[22] E. Driouch, W. Ajib and C. Assi, "Efficient Heuristics for Clustering and Power Allocation in Small Cell Networks," 2015 IEEE 82nd Vehicular Technology Conference VTC2015-Fall, Boston, MA, pp. 1-5, 2015.

[23] M. Sadeghzadeh, H. R. Bahrami, and N. H. Tran, "Clustered linear precoding for downlink network MIMO systems with partial CSI," Wireless Communications and Mobile Computing, vol. 16, no. 15, pp. 2340-2355, 2016.

[24] S. Y. Shin and T. A. Nugraha, "Effect of channel estimation error on coordinated small-cells with block diagonalization," Applied Mechanics and Materials, vol. 556-562, pp. 4501-4504, 2014.

[25] D. Ben Cheikh, J. Kelif, M. Coupechoux and P. Godlewski, "Multicellular Zero Forcing Precoding Performance in Rayleigh and Shadow Fading," 2011 IEEE 73rd Vehicular Technology Conference VTC Spring, Yokohama, pp. 1-5, 2011.

[26] Soo Young Shin and T. A. Nugraha, "Cooperative water filling (CoopWF) algorithm for small cell networks," 2013 International Conference on ICT Convergence ICTC, Jeju, pp. 959-961, 2013.

\section{BIOGRAPHIES OF AUTHORS}

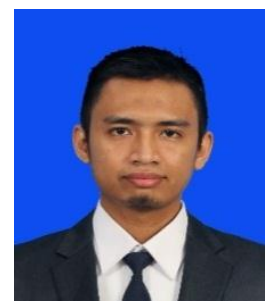

Toha Ardi Nugraha received the B.Sc. degree in Telecommunication Engineering from Telkom University, Indonesia, in 2011 and the M.Eng. degree in IT Convergence Engineering from Kumoh National Institute of Technology, South Korea, in 2014. He worked at Research Centre, PT. Telkom Indonesia for two years from 2000 to 2012, as a Research Assistant, and participated in FP7 FREEDOM project founded by European Commission. Since 2016, He joined at Department of Electrical Engineering, Universitas Muhammadiyah Yogyakarta, Indonesia, as a lecturer. Currently, he is pursuing Ph.D. degree at the Department of Telecommunication Engineering, Czech Technical University in Prague (CVUT), Czech Republic. His research interest includes Wireless and Mobile Networks, Small-Cells, Device-to-Device Communication, and Internet of Things.

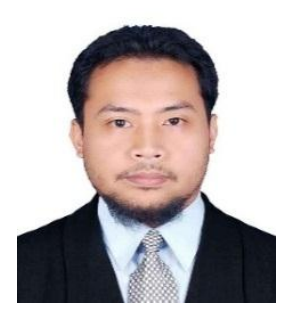

Indar Surahmat obtained Bachelor Degree from Universitas Gadjah Mada in 2005 and Master Degree from Institut Teknologi Bandung in 2011. Both are in Electrical Engineering. From 2005-2009, he worked at a telecommunication company as a Radio Frequency Network and Planning Optimization Engineer. After graduated from Master Degree, he went back to work in the same field until 2015. During work as a RF Engineer, he had handled thousands of BTS deployments and optimized hundreds of site clusters. In 2015, he joined Universitas Muhammadiyah Yogyakarta as a researcher and a lecturer. In 2017, he received a certification as a professional engineer from The Institution of Engineers Indonesia. He is currently pursuing $\mathrm{PhD}$ in Institute of High Frequency Technology, RWTH Aachen University. His research are in fields of antennas for wireless communications, cellular networks, propagation models, and traffic engineering.

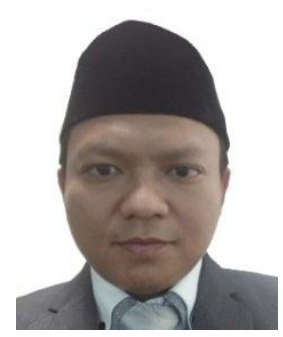

Firdaus received the B.Eng degree in Electrical Engineering from Gadjah Mada University, Yogyakarta in 2007 and M.Eng Degree in Telecommunication from Telkom University, Bandung in 2010. He worked at Department of Electrical Engineering, Universitas Islam Indonesia, Yogyakarta since 2010. He is currently pursuing his PhD in Razak Faculty of Technology and Informatics, Universiti Teknologi Malaysia, Kuala Lumpur. He just passed the thesis exam in November 2019. His research interest is in wireless communication, wireless sensor network and indoor positioning. 